\title{
Increased Asics Expression via the Camkii- CREB Pathway in a Novel Mouse Model of Trigeminal Pain
}

\author{
Yan Wang ${ }^{\mathrm{a}}$ Xiujuan Fu ${ }^{\mathrm{a}} \quad$ Lifang Huang $^{\mathrm{a}} \quad \mathrm{Xi} \mathrm{Wang}^{\mathrm{a}}$ Zuneng $\mathrm{Lu}^{\mathrm{a}}$ Fan Zhu ${ }^{\mathrm{b}}$ \\ Zheman Xiao \\ aDepartment of Neurology, Institution of Neuropsychiatry Research, Renmin Hospital of Wuhan \\ University, Wuhan, Hubei, bHubei Province Key Laboratory of Allergy and Immunology, Wuhan, China
}

\section{Key Words}

Asics • Trigeminal pain • C-FOS gene $•$ CaMKII-CREB • Migraine

\begin{abstract}
Background/Aims: Migraine is a disabling condition that severely impacts socioeconomic function and quality of life. The focus of this study was to develop a mouse model of trigeminal pain that mimics migraine. Methods: After undergoing dural cannulation surgery, mice were treated with repeated dural doses of an acidic solution to induce trigeminal pain. Results: The method elicited intermittent, head-directed wiping and scratching as well as the expression of both the c-FOS gene in the spinal trigeminal nucleus caudalis and calcitonin gene related peptide (CGRP) in the periaqueductal grey matter. Interestingly, the acid-induced trigeminal pain behaviour was inhibited by amiloride, an antagonist of acid-sensing ion channels (ASICs), but not by AMG-9810, an inhibitor of transient receptor potential cation channel V1(TRPV1). In addition, the relative mRNA and protein expression levels of ASIC1a and ASIC3 were increased in the acid-induced trigeminal nociceptive pathways. Furthermore, blocking CaMKII with KN-93 significantly reduced the acid-induced trigeminal pain behaviour and c-FOS gene expression. Conclusion: The data suggested that chronic intermittent administration of an acidic solution to mice resulted in trigeminal hypersensitivity and that dural acid-induced trigeminal pain behaviour in mice may mechanistically mimic migraine. The observations here identify an entirely novel treatment strategy for migraine.
\end{abstract}

\section{Introduction}

Migraine and other primary headache disorders are common, multifactorial, neurovascular diseases that significantly affect individuals and society. Migraine typically begins as an episodic disorder, but commonly progresses to a frequent or even daily condition

Y. Wang and X. Fu contributed equally to this work.

Zheman Xiao

KARGER
Department of Neurology, Institution of Neuropsychiatry Research,

Renmin Hospital of Wuhan University, Jiefang Road 238, Wuhan, Hubei (PR China)

Tel. +027 88041911-87982, Fax +027 88041911-87982, E-Mail zmxiao@whu.edu.cn 


\section{Cellular Physiology Cell Physiol Biochem 2018;46:568-578

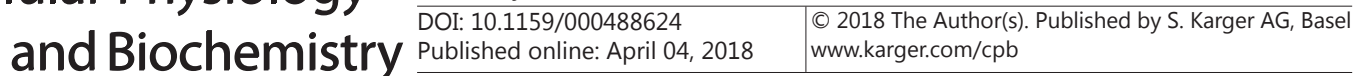

Wang et al.: Increased Asics Expression in a Novel Mouse Model of Trigeminal Pain

[1]. However, the potential pathophysiological mechanisms underlying this change are poorly understood. In addition, a lack of predictive animal models has been a significant obstacle to the identification of new migraine therapies [2]. In this study, our focus was to develop a mouse model of trigeminal pain to mimic migraine.

Dural administration of inflammatory soup (IS) is a well-established preclinical model of migraine in rats $[3,4]$. However, the composition of IS is complex, as it contains unstable, small peptides that are difficult to store. Yan et al. have previously reported a study on acute pH-evoked migraine-related pain behaviour, such as facial and hind paw allodynia [5]. An animal model with repeated activation of the dural nociceptors is needed to identify both new mechanisms of trigeminal pain and important factors involved in the transition from episodic to chronic trigeminal pain. Here, we have used acid-induced trigeminal pain to model the progression of migraine from an acute to a chronic state. We tested whether dural administration of an acidic solution caused behavioural changes in awake, freely moving mice. Furthermore, this trigeminal pain model can be applied to genetically modified mice to facilitate research on the mechanisms and therapeutic targets of migraine.

\section{Materials and Methods}

Mice

Male C57BL/6 mice (Specific Pathogen Free grade, weight 20-30 g) were housed (2-5 per cage) in the animal facility at Renmin Hospital of Wuhan University. All mice were allowed to acclimate individually for 5 days before surgery and behavioural tests. Mice were handled mildly during each habituation period until they showed no signs of freezing or rapid escaping when touched by the experimenter.

All experiments in this study were consistent with the guidelines of the Committee for Research and Ethical Issues of the Renmin Hospital of Wuhan University. All procedures were approved by the Animal Studies Committee at Ren min Hospital of Wuhan University.

\section{Dural Cannulation surgery [3, 4]}

Mice were anaesthetized with $10 \mathrm{mg} / \mathrm{mL}$ pentobarbital sodium $(0.01 \mathrm{~mL} / \mathrm{g})$. After the mice were anaesthetized, the skull was exposed via a longitudinal incision along the sagittal suture. A $1 \mathrm{~mm}$ hole in the middle of the superior sagittal sinus (between bregma and lambda) was made with a precooled dental drill (DH-0 Pin Vise, Plastics One) to carefully expose the dura. A guide cannula (22 GA, \#C313G, Plastics One), designed to extend $0.5 \mathrm{~mm}$ from the pedestal to avoid irritation of the dural tissue, was inserted into the hole and sealed into place with a mixture of dental cement powder (Stoelting 51459) and superglue. A dummy cannula (\#C313DC, Plastics One Inc.) was inserted to ensure patency of the guide cannula. Subsequently, the skin incision was closed with a silk suture. After surgery, C57BL/6 mice were allowed to recover from the surgery and anaesthesia for 5 days before the effects of dural acidic solution administration were tested.

\section{Dural administration and behavioural testing}

To establish the acid-induced recurrent trigeminal pain model, C57BL/6 mice were randomly divided into four groups: the sham group, the negative control group ( $\mathrm{pH}=7.4$ synthetic interstitial fluid, $\mathrm{pH}$ 7.4 SIF), the experimental group ( $\mathrm{pH}=6.0$ synthetic interstitial fluid, $\mathrm{pH} 6.0 \mathrm{SIF}$ ), and the positive control group (IS group). Six mice were assigned to each group. The SIF consisted of $10 \mathrm{mM} \mathrm{HEPES,} 5 \mathrm{mM} \mathrm{KCl,} 1 \mathrm{mM} \mathrm{MgCl}$, $2.5 \mathrm{mM} \mathrm{CaCl}_{2}$, and $135 \mathrm{mM} \mathrm{NaCl}$ (adjusted to $\mathrm{pH} \mathrm{7.4).} \mathrm{The} \mathrm{IS} \mathrm{contained} 1 \mathrm{mM}$ histamine, serotonin, and bradykinin and $0.1 \mathrm{mM}$ prostaglandin $\mathrm{E}_{2}$ in $\mathrm{pH}$ 7.4 SIF $[5,6]$. Sham mice were habituated to the test room and recording cage with the same protocol as the mice in other groups but were not subjected to drug administration. We administered $10 \mu$ l of vehicle (pH 7.4 SIF or IS) or acidic solution ( $\mathrm{pH} 6.0$ SIF) to the dura every other day over sixteen days (totalling eight doses), and behavioural observations occurred after the last dose. Immediately following the last dosing, mice were returned to a clean cage, their behaviours were recorded by digital video cameras for 2 hours, and then the mice were euthanized. The entire 2-hour long video was watched and scored. The time spent on unilateral forepaw wiping and hind paw scratching of the V1 dermatome (including the scalp and periorbital area) was quantified as nocifensive behaviour. Bilateral rubs were not included because they are considered normal grooming behaviour. 


\section{Cellular Physiology Cell Physiol Biochem 2018;46:568-578

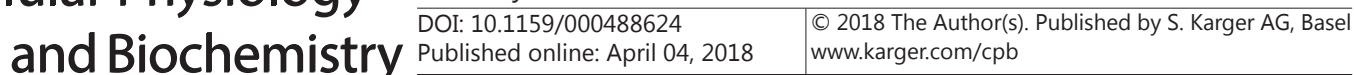 \\ Wang et al.: Increased Asics Expression in a Novel Mouse Model of Trigeminal Pain}

To explore the pathophysiology of acid-induced hyperalgesia, we intermittently co-administered $\mathrm{pH}$ 6.0 SIF with amiloride, AMG-9810 or KN-93 to the dura of pre-treated mice. Amiloride, KN-93 and AMG9810 were prepared as described by Yan et al [5].. The behavioural observation method was the same as the above. All testing was performed in a blinded manner with respect to group assignment.

\section{Immunohistochemistry}

Mice were euthanized with pentobarbital sodium. The brainstem and upper cervical spinal cord (to C3) were perfused with phosphate-buffered saline (PBS, pH 7.4) and fixed by perfusion with 4\% paraformaldehyde by standard immunohistochemical methods [7, 8]. Sections (20 $\mu \mathrm{m}$ thick) were cut through the midbrain at the PAG or caudal medulla level and through the upper cervical spinal cord at the Sp5C level. Sections were blocked in 4\% BSA/0.3\% Triton X-100 in PBS 10 mM, stained with rabbit antic-FOS (1:100, Santa Cruz) or mouse anti-CGRP (1:100, Abcam) antibodies, diluted in blocking solution for $48 \mathrm{~h}$ at $4{ }^{\circ} \mathrm{C}$ and incubated with anti-rabbit secondary antibody diluted in PBS for $2 \mathrm{~h}$ at room temperature. Detection was performed with the ABC kit (Vector) using 3, 3'-diaminobenzidine (DAB) (Sigma Aldrich) as the peroxidase substrate. The number of neurons in the Sp5C containing c-FOS or in the PAG containing CGRP was quantified under a 10x objective on a Nikon TE2000S epifluorescence microscope by a single observer blinded to the experimental treatments.

\section{Quantitative real-time PCR}

Total RNA was extracted from TG using a one-step method according to the instructions for TRIzol@ reagent (Invitrogen, USA). cDNA was then synthesized using reverse transcription in accordance with the kit instructions (BioTeke). Mouse primer sequences were as follows: GAPDH (forward 5'-GTTCCTACCCCCAATGTGTCC-3', reverse 5'-TAGCCCAAGATGCCCTTCAGT-3', 125 bp product); ASIC1a (forward 5'-TGGGGAGAGACTGATGAGACAT-3', reverse 5'-CTCCTGGCAAGACACAAACG-3', 135 bp product); and ASIC3 (forward 5'-ACTGTTTATCGGAGCCAGCC-3', reverse 5'-CTTCCTGGAGCAGAGTGTTG-3', 140 bp product). PCR amplification of cDNA was performed using a Quantitect SYBR Green PCR Kit (Roche, USA) as previously described [9]. Relative gene expression was calculated using the $2^{-\triangle \triangle \mathrm{CT}}$ method.

\section{Western blotting}

Protein samples from the trigeminocervical complex were separated on SDS-PAGE gels and transferred to PVDF membranes. The membranes were incubated overnight at $4^{\circ} \mathrm{C}$ with primary anti-ASIC1a $(1: 100$; Alomone Labs), anti-ASIC3 (1:100; Alomone Labs), anti-phosphoThr-286-CaMKII monoclonal antibody (1:200; Abcam), anti-CaMKII polyclonal antibody (1:200; Abcam), or anti-GAPDH (1:1000; Abcam) antibodies and finally were visualized using an enhanced chemiluminescence system (UVPLabWorks, CA). Signal intensity was measured using Image software (NIH, USA) for the analysis of ASIC1a, ASIC3, phosphoCaMKII (p-CaMKII), and CaMKII expression in the TCC [10].

\section{Statistical analysis}

All data are presented as the means \pm SD. The Shapiro-Wilk test was used to check data normality. Statistical analyses were assessed by SPSS 19.0 software, using Student's t-test or analysis of variance (ANOVA) with post hoc analysis where appropriate. A value of $p<0.05$ was considered statistically significant.

\section{Results}

Acid-induced recurrent trigeminal pain

Repeated administration of an acidic solution elicits trigeminal V1 dermatome nociceptive behaviour in mice. The acute administration of an acidic solution $(\mathrm{pH}=6.0$ synthetic interstitial fluid, pH 6.0 SIF) was previously shown to evoke acute facial and hind paw allodynia [5]. To mimic the progression of recurrent trigeminal pain, an acidic solution was administered every other day for sixteen days, totalling eight doses of acidic solution (Fig. 1A). First, we allowed mice to recover from surgery for 5 days before testing the effects of dural administration of either an acidic solution ( $\mathrm{pH} 6.0$ SIF) or vehicle $(\mathrm{pH}=7.4$ synthetic interstitial fluid, pH 7.4 SIF or IS) on behaviour. We found no difference in the behaviour 
between the naive and surgically intervened mice. Thus, the risk of the catheter surgery affecting mouse behaviour was negligible. During the 2 hours after the administration of the acidic solution, digital video cameras recorded behavioural observations including forepaw wiping and hind paw scratching around the scalp and periorbital area within the trigeminal V1 dermatome. The duration of each of these behaviours was quantified separately for each group.

Compared with the pH 7.4 SIF treatment, repetitive intermittent $\mathrm{pH} 6.0$ SIF administration over a 16- day period elicited significant chronic trigeminal pain. The maximum effect occurred approximately 30-40 min after pH 6.0 SIF administration. Mice in the dural pH 6.0 SIF treatment group spent significantly more time wiping and scratching than the negative control pH 7.4 SIF-treated mice (Fig. 1B). However, there were no significant differences between mice treated with pH 6.0 SIF and the positive control IS-treated mice (Fig. 1B).

Fig. 1. Episodic dural administration of an acidic solution elicits trigeminal V1 dermatome nociceptive behaviour in adult male mice. (A) Time axis of experiments. (B) Total duration of forepaw wiping and hind paw scratching in response to dural administration of the negative control solution ( $\mathrm{pH}=7.4$ synthetic interstitial fluid, pH 7.4 SIF), the experimental solution $\quad(\mathrm{pH}=6.0 \quad$ synthetic interstitial fluid, $\mathrm{pH} \quad 6.0$ SIF), and the positive control solution (inflammatory soup, IS) and shamtreated mice during the 2 hours recording period, respectively. ( $\mathrm{n}=6$ in each group) $\left({ }^{*} \mathrm{p}<0.001\right.$, t-test between the pH 6.0 and $\mathrm{pH}$ 7.4 groups); however, there were no significant differences between mice treated with pH 6.0 SIF and the positive control IS-treated mice. (C)The each of duration of forepaw wiping and hind paw scratching in response to dural administration of the negative control solution $(\mathrm{pH}=7.4$ synthetic interstitial fluid, $\mathrm{pH} 7.4 \mathrm{SIF}$ ), the
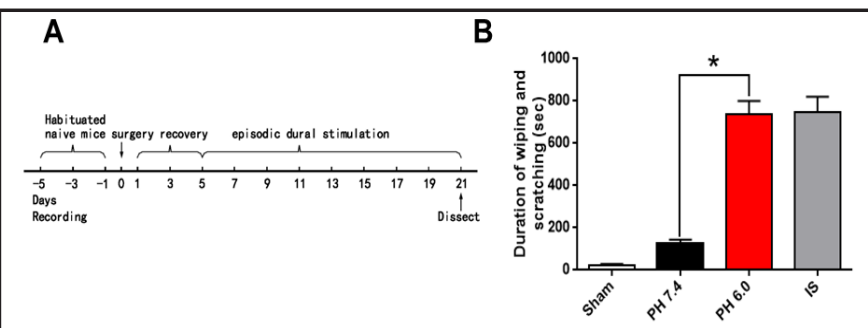

C

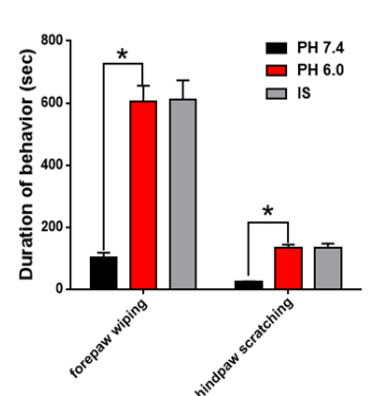

E

D

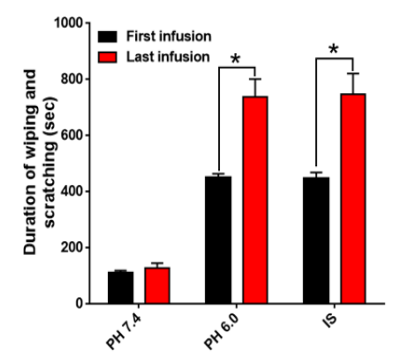

$\mathbf{F}$

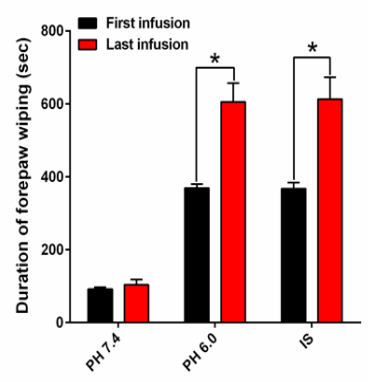

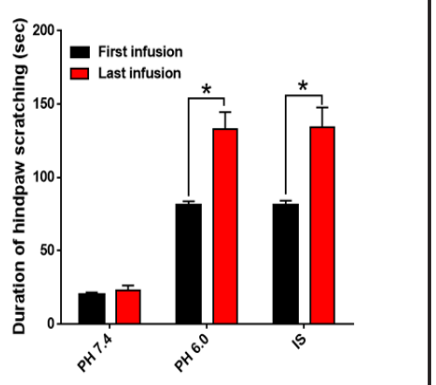

experimental solution ( $\mathrm{pH}=6.0$ synthetic interstitial fluid, $\mathrm{pH} 6.0 \mathrm{SIF}$ ), and the positive control solution (inflammatory soup, IS) during the 2 hours recording period, respectively. ( $n=6$ in each group) $\left({ }^{*} p<0.001\right.$, t-test between the $\mathrm{pH} 6.0$ and $\mathrm{pH} 7.4$ groups); however, there were no significant differences between mice treated with pH 6.0 SIF and the positive control IS-treated mice. (D) Total duration of forepaw wiping and hind paw scratching in response to dural administration of the negative control solution ( $\mathrm{pH}=7.4$ synthetic interstitial fluid, pH 7.4 SIF), the experimental solution ( $\mathrm{pH}=6.0$ synthetic interstitial fluid, $\mathrm{pH} 6.0$ SIF), and the positive control solution (inflammatory soup, IS) after the first and the last infusion, respectively. ( $\mathrm{n}=6$ in each group) $\left({ }^{*} \mathrm{p}<0.001\right.$, t-test between the first and last infusion). (E) (F) The each of duration of forepaw wiping and hind paw scratching in response to dural administration of the negative control solution $(\mathrm{pH}=7.4$ synthetic interstitial fluid, $\mathrm{pH} 7.4 \mathrm{SIF}$ ), the experimental solution ( $\mathrm{pH}=6.0$ synthetic interstitial fluid, $\mathrm{pH}$ 6.0 SIF), and the positive control solution (inflammatory soup, IS) after the first and the last infusion, respectively. ( $n=6$ in each group) $(* p<0.001$, t-test between the first and last infusion). 


\section{Cellular Physiology Cell Physiol Biochem 2018;46:568-578 \begin{tabular}{c|c|c|} 
DOI: 10.1159/000488624 & O 2018 The Author(s). Published by S. Karger AG, Basel \\
wwww.karger.com/cpb
\end{tabular} \\ Wang et al.: Increased Asics Expression in a Novel Mouse Model of Trigeminal Pain}

Moreover, we separately analysed forepaw wiping and hind paw scratching. Both forepaw wiping and hindpaw scratching around the scalp and periorbital area were significantly more robust in mice that received dural administration of pH 6.0 SIF than in pH 7.4 SIFtreated mice (Fig. 1C).

To measure the transition from episodic to chronic trigeminal pain, the duration of the behaviours after the first and last infusion was quantified separately for each group. Compared with the extent of forepaw wiping and hind paw scratching after the first infusion, these behaviours were significantly more robust in mice after the last infusion with pH 6.0 SIF (Fig. 1D, E, F). However, no significant differences were found between the first and last infusion in mice treated with pH 7.4 SIF (Fig. 1D, E, F).

Dural administration of an acidic solution alone induces c-FOS protein expression in the spinal trigeminal nucleus caudalis (Sp5C). Previous studies have shown that the protein expression of c-FOS, an immediate early gene product, in Sp5C neurons which receives nociceptive input, can be used as a marker of neuronal activation of the trigeminovascular pathway [11]. We recorded the behaviour of pH 7.4 SIF-, pH 6.0 SIF-, IS- and sham-treated mice for 2 hours and then quantified c-FOS expression in Sp5C neurons of the mice after the last infusion. Compared with the number of c-FOS-positive neurons in the pH 7.4 SIF-treated group, the number these neurons in the pH 6.0 SIF-treated mice were increased 8.6 fold in the Sp5C (Fig. 2A, B). By contrast, there was no statistical association between the pH 6.0 SIF-treated group and the positive control IS-treated group (Fig. 2A, B).

Dural administration of an acidic solution alone induces robust calcitonin gene related peptide (CGRP) expression in the periaqueductal grey (PAG). The contribution of the neuropeptide CGRP to migraine pathophysiology is well-established, and CGRP receptor antagonists have been effective for treating migraine in clinical trials [12]. Similarly, we recorded the nocifensive behaviour of the four treatment groups for 2 hours and then quantified CGRP expression in the PAG neurons of the same mice after the last infusion. The number of CGRP-positive neurons was significantly greater in the $\mathrm{pH} 6.0$ SIF-treated group than in the negative control pH 7.4 SIF-treated group (Fig. 2C, D). However, no significant difference in the number of CGRP-positive neurons was observed between the pH 6.0 SIF-treated group and the positive control IS-treated group (Fig. 2C, D).

These results suggested that dural administration of pH 6.0 SIF can mimic not only the trigeminal V1-directed trigeminal pain behaviour but also c-FOS and CGRP expression

Fig. 2. Dural administration of an acidic solution alone induces pain related protein expression. (A) (B) Representative images of c-FOS in spinal trigeminal nucleus caudalis (Sp5C) in mice that received vehicle $(\mathrm{pH} 7.4 \mathrm{SIF}$ or IS) and pH 6.0 SIF after the last infusion. ( $\mathrm{n}=6$ in each group) (The area shown in red square is the target Sp5C) $\left({ }^{*} \mathrm{p}<0.001\right.$, t-test between the $\mathrm{pH} 6.0$ and $\mathrm{pH} 7.4$ groups); there was no statistical differences between pH 6.0 and IS groups. (C)(D) Representative images of calcitonin gene related

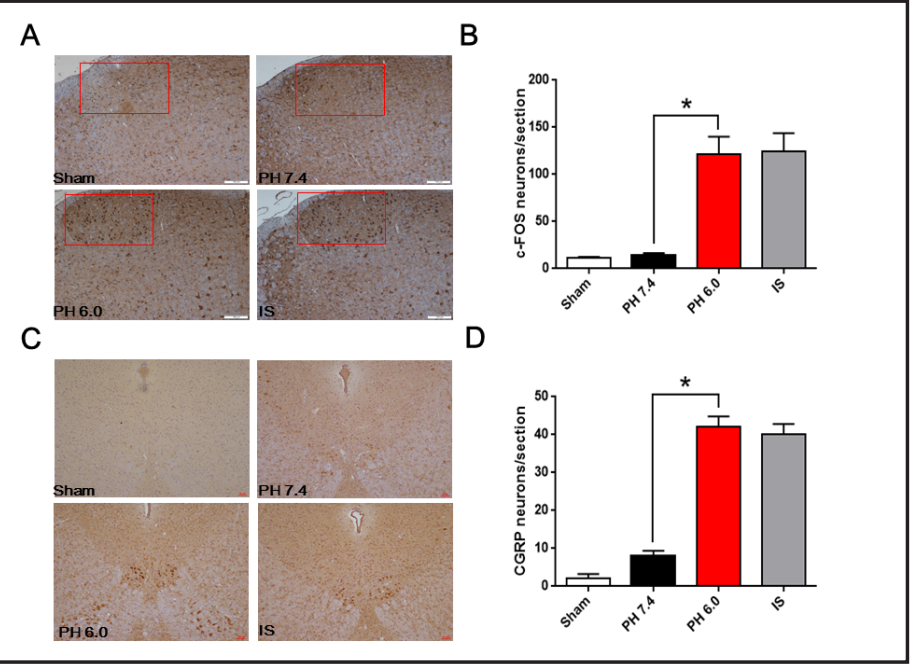
peptide(CGRP) in periaqueductal gray (PAG) in mice that received vehicle (pH 7.4 SIF or IS) and pH 6.0 SIF after the last infusion. ( $\mathrm{n}=6 \mathrm{in}$ each group) $\left({ }^{*} \mathrm{p}<0.001\right.$, t-test between the $\mathrm{pH} 6.0$ and $\mathrm{pH} 7.4$ groups); however, there was no statistical differences between $\mathrm{pH} 6.0$ and IS groups. 


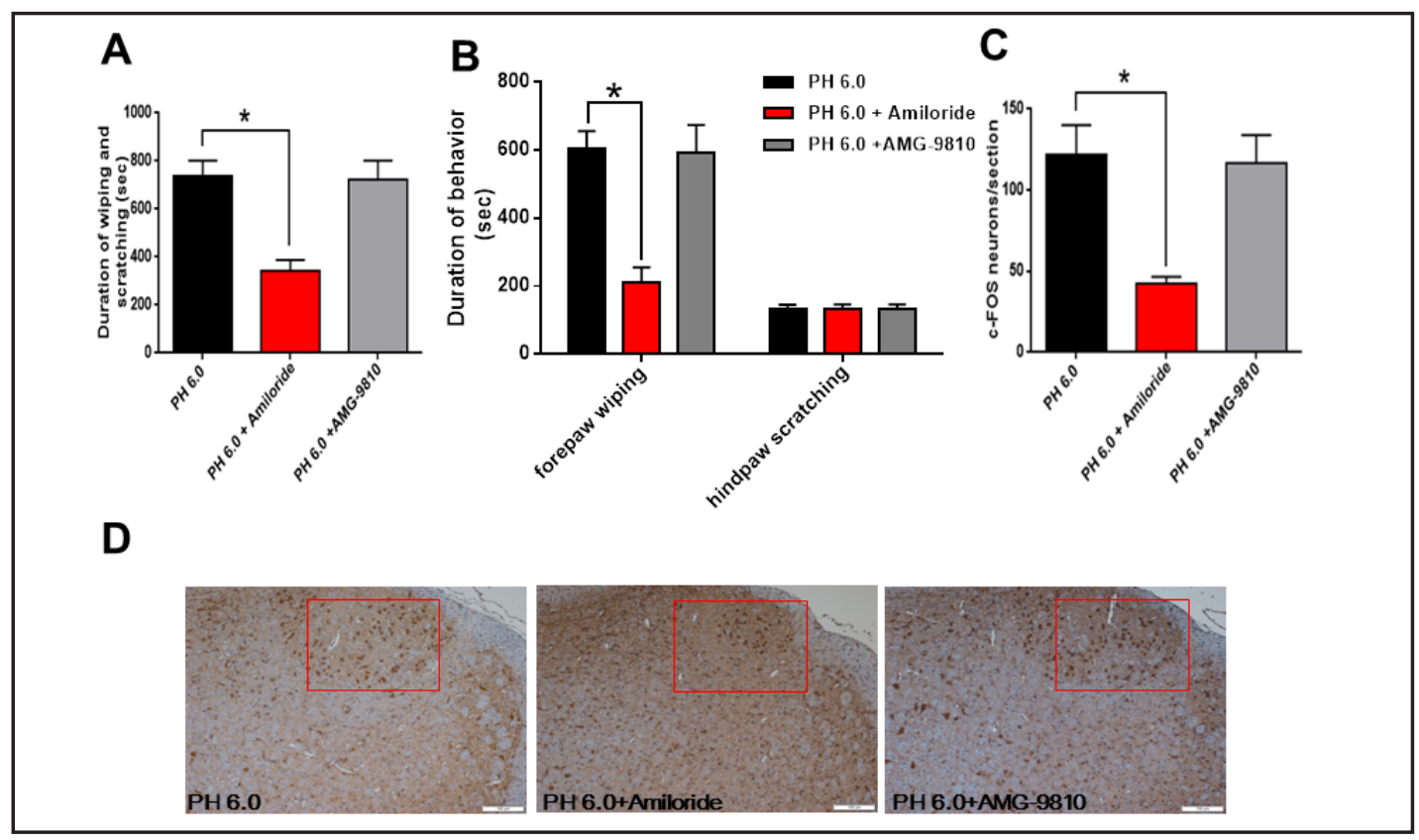

Fig. 3. Dural acid-induced trigeminal pain and c-FOS expression in Sp5C were blocked by amiloride, antagonist of acid-sensing ion channel (ASICs), not by AMG9810. (A) Total duration of forepaw wiping and hind paw scratching in response to dural application of pH 6.0 SIF alone, co-application of amiloride or AMG9810 with pH 6.0 SIF during the 2 hours recording period, respectively. Co-application of amiloride with $\mathrm{pH}$ 6.0 SIF significantly shorten the total duration of forepaw wiping and hind paw scratching ( $\mathrm{n}=6$ in each group) $\left({ }^{*} \mathrm{p}<0.001\right.$, t-test between the amiloride and $\mathrm{pH} 6.0$ group); however, there was no statistical differences between the AMG9810 and pH 6.0 group. (B) Co-application of amiloride with pH 6.0 SIF significantly blocked the behavior of forepaw wiping $\left({ }^{*} \mathrm{p}<0.001\right.$, t-test between the amiloride and $\mathrm{pH} 6.0$ group), while it had no difference on the behavior of hand paw scratching. Moreover, there was no statistical difference between the AMG9810 and pH 6.0 group in both forepaw wiping and hind paw scratching. (C) (D) Representative images of c-FOS in spinal trigeminal nucleus caudalis (Sp5C) in mice that received pH 6.0 SIF and co-treatment amiloride with pH 6.0 SIF after the last infusion. Co-application of amiloride with pH 6.0 SIF significantly blocked c-FOS expression in Sp5C but co-treatment AMG-9810 with pH 6.0 SIF failed to blocked c-FOS expression in Sp5C. ( $\mathrm{n}=6$ in each group) (The area shown in red square is the target Sp5C) $\left({ }^{*} \mathrm{p}<0.001\right.$, t-test between the amiloride and $\mathrm{pH} 6.0$ group); however, there was no statistical differences between the AMG9810 and $\mathrm{pH} 6.0$ group.

induced by IS administration in mice. Therefore, we established a preliminary model of acidinduced recurrent trigeminal pain.

Nocifensive behaviour and c-FOS expression in the Sp5C of dural acid-induced mice were decreased by an antagonist of ASICS but not of transient receptor potential cation channel V1 (TRPV1)

Acidic solutions have been shown to activate both ASICs and TRPV1 [13]. We wondered whether dural acid treatment induced trigeminal pain in mice through ASICs or TRPV1. To address this question, we assessed acid-induced nocifensive behaviour in mice treated with either amiloride (an ASICs antagonist) or AMG-9810 (a TRPV1 antagonist). We quantified the duration of both forepaw wiping and hind paw scratching as well as the expression of c-FOS expression in the Sp5C. Co-treatment of AMG-9810 did not alter the dural acid-induced trigeminal pain behaviour. In contrast, co-treatment with amiloride blocked the nocifensive behaviour elicited by dural administration of pH 6.0 SIF alone. Mice co-treated with amiloride generally presented a shorter duration of nocifensive behaviour than mice treated with $\mathrm{pH}$ 6.0 SIF alone (Fig. 3A). Similarly, we also quantified the duration of forepaw wiping and hind paw scratching separately. Compared with mice treated with pH 6.0 SIF alone, mice KARGER 
co-treated with amiloride and pH 6.0 SIF presented significantly less robust forepaw wiping (Fig. 3B). However, amiloride had no effect on hind paw scratching. Moreover, there was no significant difference in the total duration of forepaw wiping and hind paw scratching between the mice co-treated with AMG-9810 and mice treated with pH 6.0 SIF alone (Fig. $3 \mathrm{~A}, \mathrm{~B})$.

In addition, dural co-administration of amiloride resulted in a 2.8-fold decrease in the number of c-FOS-positive Sp5C neurons in mice compared with administration of pH 6.0 SIF alone (Fig. 3C, D). In contrast, there was no significant difference between the mice cotreated with AMG-9810 and the mice treated with pH 6.0 SIF alone (Fig. 3C, D).

Consequently, daily episodic treatment with amiloride significantly inhibited the development of trigeminal pain, induced by the acidic solution treatment. ASICs might play a central role in regulating acid-induced recurrent trigeminal pain.

\section{Upregulation of ASICs in the trigeminal nociceptive pathways}

To further verify the expression of ASICs in the trigeminal nociceptive pathways, the trigeminal ganglion (TG) and trigeminocervical complex (TCC) were dissected to detect mRNA and protein expression levels. Compared with those of the pH 7.4 SIF-treated group, the relative mRNA expression levels of ASIC1a and ASIC3 in the TG of the pH 6.0 SIF-treated group were increased 1.59- and 1.65-fold, respectively (Fig. 4A, B). Moreover, compared with the pH 7.4 SIF-treated group, the pH 6.0 SIF-treated group showed 1.67- and 1.74-fold increases in the relative protein expression levels of ASIC1a and ASIC3, respectively, in the TCC (Fig. 4C, D, E, F). These data further validate that ASICs play an important role in acidinduced trigeminal pain.

\section{Inhibition of CaMKII phosphorylation blocked acid-induced trigeminal pain}

We have shown the robust activation of ASICs in the acid-induced trigeminal nociceptive pathways, but the downstream pain-modulating pathway that induces c-FOS expression in the Sp5C is still unknown. Activation of the CaMKII/CREB signalling pathways in primary afferent neurons has been implicated in nociceptive sensitization under various persistent

Fig. 4. Increased of the mRNA and protein expression level of ASICs in trigeminal nociceptive pathways in acid-induced trigeminal pain. (A) (B) Quantitative analysis of mRNA expression of ASIC1a or ASIC3 in trigeminal ganglion between $\mathrm{pH}$ 6.0 SIF and pH 7.4 SIF group. Both the mRNA expression of ASIC1a and ASIC3 were increased in $\mathrm{pH}$ 6.0 SIF compared with $\mathrm{pH}$ 7.4 SIF group $\left({ }^{*} \mathrm{p}<0.001\right.$, t-test between the $\mathrm{pH} 6.0$ and $\mathrm{pH} 7.4$ group). (C) (D) (E) (F) Western blotting analysis of ASIC1a or ASIC3 in trigeminocervical complex in $\mathrm{pH}$ 6.0 SIF and pH 7.4 SIF group. Both the protein expression of ASIC1a and ASIC3 were increased in $\mathrm{pH}$ 6.0 SIF compared with $\mathrm{pH}$ 7.4 SIF group $\left({ }^{*} \mathrm{p}<0.05\right.$, t-test between the pH 6.0 and pH 7.4 group).

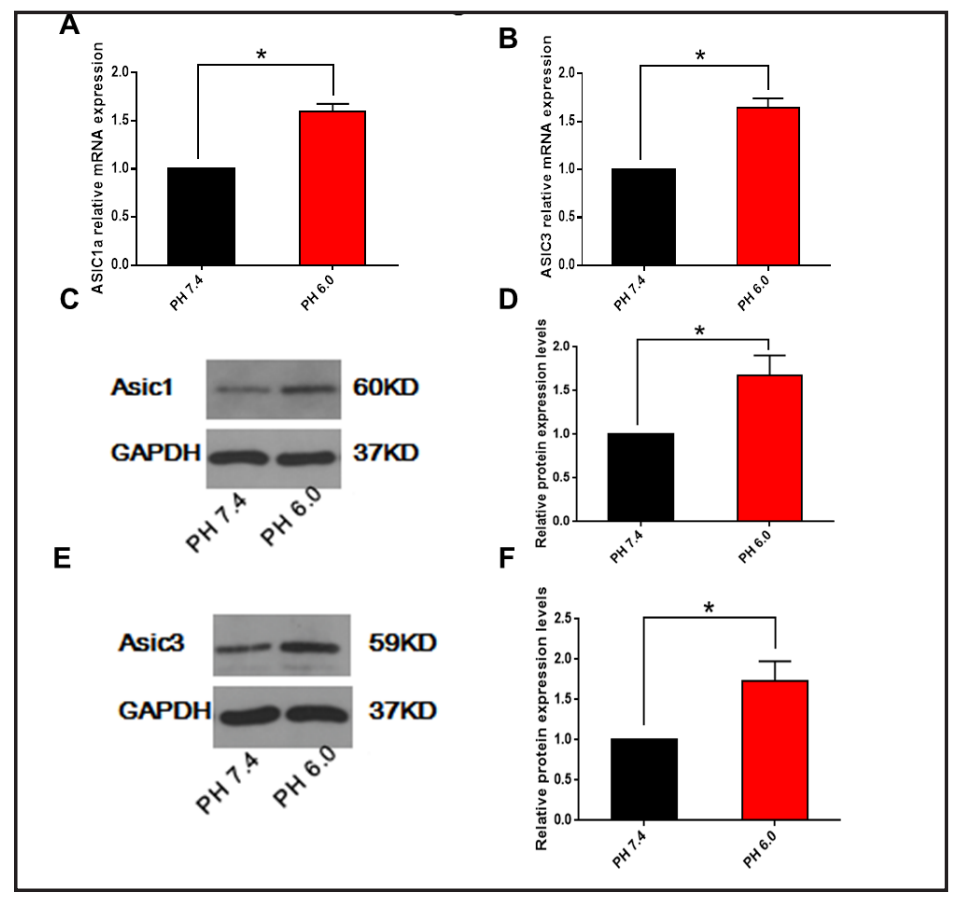


Fig. 5. CaMKII phosphorylate contributes to dural acid-induced trigeminal pain. (A) Total duration of forepaw wiping and hind paw scratching in response to dural application of $\mathrm{pH} 6.0 \mathrm{SIF}$ alone, co-application of $\mathrm{KN}-93$ with $\mathrm{pH}$ 6.0 SIF or pH 7.4 SIF alone during the 2 hours recording period, respectively. Co-application of KN-93 with pH 6.0 SIF obviously shorten the total duration of forepaw wiping and hind paw scratching ( $\mathrm{n}=6$ in each group) $\left({ }^{*} \mathrm{p}<0.001\right.$, t-test between the KN93 and $\mathrm{pH} 6.0$ group; $\# \mathrm{p}<0.001$, t-test between the $\mathrm{pH} 6.0$ and $\mathrm{pH}$ 7.4 group). (B) Co-application of KN-93 with pH 6.0 SIF obviously blocked the behavior of forepaw wiping $\left({ }^{*} \mathrm{p}<0.001\right.$, t-test between the $\mathrm{pH} 6.0$ and $\mathrm{KN}-93$ group), while it had no effect on the behavior of hand paw scratching. (C) (D)

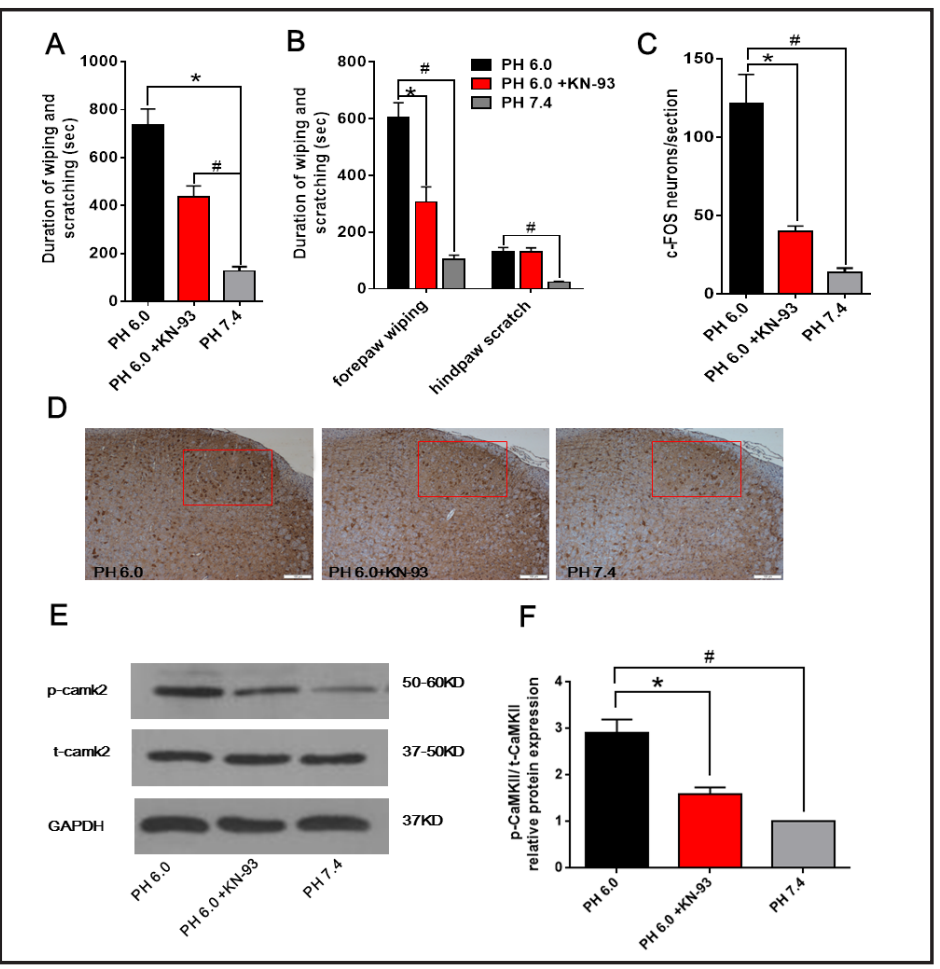
Representative images of c-FOS in spinal trigeminal nucleus caudalis (Sp5C) in mice that received pH 6.0 SIF and co-treatment KN-93 with pH-6.0 SIF after the last infusion. Co-application of KN-93 with pH 6.0 SIF obviously blocked c-FOS expression in Sp5C. ( $n=6$ in each group) (The area shown in red square is the target Sp5C) $\left({ }^{*} \mathrm{p}<0.001\right.$, t-test between the pH 6.0 and $\mathrm{KN}-93$ group; $\# \mathrm{p}<0.001$, t-test between the $\mathrm{pH}$ 6.0 and pH 7.4 group). (E) (F) Western blotting analysis of phospho-CaMKII (p-CaMKII) and total CaMKII (t-CaMKII) of trigeminocervical complex in pH 6.0 SIF alone, co-application of KN-93 with pH 6.0 SIF or pH 7.4 SIF alone. The relative expression level of p-CaMKII/ $t$-CaMKII in each group is as (F). The proportion of active CaMKII (p-CaMKII/ $\mathrm{t}$-CaMKII) in the pH 6.0 SIF-treated group was increased compared with that in the pH 7.4 SIF-treated group $\left({ }^{*} \mathrm{p}<0.001\right.$, t-test between the $\mathrm{KN}-93$ and $\mathrm{pH} 6.0$ group; \#p<0.001, t-test between the $\mathrm{pH} 6.0$ and $\mathrm{pH} 7.4$ group).

pain conditions, including migraine [14]. We tested the effect of KN-93, an inhibitor of CaMKII, on acid-induced recurrent trigeminal pain. We quantified the durations of forepaw wiping and hind paw scratching separately in mice co-treated with KN-93 and pH 6.0 SIF and the mice treated with pH 6.0 SIF alone. Compared with the pH 6.0 SIF treatment alone ,cotreatment with KN-93 attenuated the nocifensive behaviour (Fig. 5A). Only forepaw wiping was significantly less robust in mice co-treated with KN-93 than mice treated with pH 6.0 SIF alone and KN-93 had no effect on hind paw scratching (Fig. 5B).Moreover, co-treatment with KN-93 also attenuated c-FOS expression, the expression of c-FOS in the Sp5C in the KN-93 group was significantly lower than that in the pH 6.0 SIF alone group (Fig. 5C, D).

In addition, immunoblotting for TCC in protein extracts further demonstrated that, despite the lack of change in total CaMKII expression between the pH 6.0 SIF- and pH 7.4 SIF-treated groups (Fig. 5E), the proportion of active CaMKII (T286-phosphorylated) in the pH 6.0 SIF-treated group was increased by 2.9-fold compared with that in the pH 7.4 SIFtreated group (Fig. 5E, F). In brief, these results showed that blocking CaMKII with KN93 significantly reduced acid-induced trigeminal pain and c-FOS expression, suggesting that the activation of CaMKII/CREB signalling pathways is necessary for the manifestation of dural acid-induced trigeminal pain. 


\section{Cellular Physiology Cell Physiol Biochem 2018;46:568-578 \\ \begin{tabular}{l|l} 
and Biochemistry Published onIIne: AprI 04, 2018 & $\begin{array}{l}\text { (c) } 2018 \text { The Author(s). Published by S. Karger AG, Basel } \\
\text { www.karger.com/cpb }\end{array}$ \\
\hline
\end{tabular} \\ Wang et al.: Increased Asics Expression in a Novel Mouse Model of Trigeminal Pain}

\section{Discussion}

Migraine and other primary headache disorders are diseases that have a greatly detrimental influence on a patient's life, as they are very serious conditions that can be incapacitating but receive little attention [1]. The clinical manifestation of migraine is believed to be determined by both genetic factors and non-genetic risk-modulating factors, which contribute to the threshold for the generation of migraine attack $[2,15,16]$. Thus, establishing suitable animal models to study migraine pathogenesis is of great significance. Pathophysiological studies have suggested that patients with recurrent migraine headache experience repeated dural nociceptor activation [2,17]. To date, no reports have been published that use repeated acidic dural stimulation to evoke recurrent trigeminal pain to mimic migraine in mice. We show that episodic stimulation of dura mater with acidic solution alone noticeably enhanced vigorous forepaw wiping and hind paw scratching behaviour in our model. Shimada et al. demonstrated that forepaw wiping is associated with trigeminal pain, but that hind paw scratching is associated with itch [18]. Thus, we separately reported unilateral forepaw wiping and hind paw scratching. Both forepaw wiping and hind paw scratching were significantly more robust in mice treated with pH 6.0 SIF than in those treated with pH 7.4 SIF. The duration of forepaw wiping was not significantly different between the acid-induced group and the positive control IS group in this study. Moreover, the protein expression of c-FOS in the Sp5C of the acid-induced group was equivalent to that of the IS group; CGRP expression in the PAG was also equal between these two groups.

In our model, $\mathrm{C} 57 \mathrm{Bl} / 6 \mathrm{~J}$ mice received dural doses of acidic solution every other day for 16 days to active the trigeminovascular system. Upon activation of these perivascular trigeminal afferents, the signal travels through the TG to neurons in the TCC. The signals are then transferred to higher brain structures, including the thalamus and cortex. Activity is modulated through the extensive connections with brainstem regions such as the PAG and the locus coeruleus $[19,20]$. The hyperactivity produced by repeated episodic trigeminal nociceptive activation is clearly different than that produced by a single short stimulus. The definition of chronic migraine by the International Classification of Headache Disorders ( $3^{\text {rd }}$ edition, ICHD-3) is the occurrence of a headache (tension-type-like and/or migrainelike) $\geqslant 15$ days per month [1]. The model in this study is consistent with the definition, as mice received intermittent dural stimulation with acid for 16 days. Many scholars have shown that IS administration can produce a rat migraine model $[3,4,7]$. Compared with IS stimulation, administration of an acidic solution alone can also induce robust trigeminal pain and transcriptional expression of c-FOS and the neurotransmitter CGRP. In summary, we explored a novel mouse model of trigeminal pain induced by repeated dural acid stimulation that mimicked migraine.

The model has the following advantages. First, a mouse model of trigeminal pain with a C57BL6 background lays the foundation for later studies with knockout or knock-in mice, as transgenic mice are widely used in the study of migraine. Second, we minimized the effects of surgery and anaesthesia by letting the mice recover from surgery for 5 days before the administration of the acid solution. Third, the acidic solution is more stable than IS, which contains small, easily degradable peptides [3, 4]. Fourth, we used dural, episodic stimulations in awake, freely moving mice to eliminate the effect of anaesthesia or surgery on the behavioural observations. Finally, an animal model of repeated dural nociceptor activation will facilitate the study of the physiological changes that occur in chronic migraine.

Dural co-administration of the ASICs antagonist amiloride effectively inhibited the acid-induced behaviour, but the specific TRPV1 antagonist AMG-9810 failed to impact the behaviour, suggesting that ASICs may be related to the on-going trigeminal pain in this model. Moreover, the relative mRNA or protein expression levels of ASIC1a and ASIC3 were increased in acid-induced trigeminal nociceptive pathways. Our results agree with the findings of Holland et al., who reported that amiloride is efficacious in the treatment of aura and headache in migraine patients with aura via an ASIC1a mechanism [19]. Deval et al. identified that ASIC3 plays a primary role in acid-induced cutaneous allodynia and migraine- 


\section{Cellular Physiology Cell Physiol Biochem 2018;46:568-578

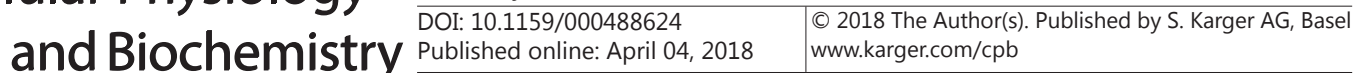

Wang et al.: Increased Asics Expression in a Novel Mouse Model of Trigeminal Pain

related pain behaviour [21]. These data further validate ASICs as a key element in the acidinduced dural afferent activation and trigeminal pain. Furthermore, cortical spreading depression has been shown to be accompanied by dural ischaemia, which can produce a drop in dural $\mathrm{pH}$ to activate ASICs and initiate signalling in migraine $[19,22]$.

Moreover, activation of the CaMKII/CREB signalling pathway is necessary for the acidinduced mouse behaviour, as evidenced by the inhibitory effect of systemically administered $\mathrm{KN}-93$. It is possible that increased $\mathrm{Ca}^{2+}$ influx via activation of ASICs may regulate many intracellular $\mathrm{Ca}^{2+}$-dependent signalling pathways by altering the physiological function of multiple $\mathrm{Ca}^{2+}$ binding proteins, amongst which CaMKII is a likely candidate $[23,24]$. Phosphorylation of CaMKII on Thr286 is essential for CaMKII activation. CaMKII inhibitors can ameliorate many types of hyperalgesia such as capsaicin-induced pain, inflammatory pain and neuropathic hyperalgesia $[14,24]$. Although total CaMKII protein expression was not altered by dural administration of acidic solution, the phospho-CaMKII protein levels were significantly increased in the model mice. In addition, treatment with the CaMKII inhibitor decreased the trigeminal pain induced by the acidic solution. Studies have shown that CaMKII can induce CREB phosphorylation in various types of pain models $[10,14$, $25,26]$. The nociceptive transcription factor CREB is essential for the regulation of pronociceptive genes, such as c-FOS. In our study, the inhibition of CaMKII significantly blocked the increase in the gene expression of c-FOS induced by the acidic solution.

\section{Conclusion}

Chronic intermittent treatment with acidic solution alone produces chronic hypersensitivity that represents a model of trigeminal pain. ASIC1a and ASIC3 levels are significantly elevated in acid-induced trigeminal nociceptive pathways, which contribute to the development of recurrent trigeminal pain through the activation of the CaMKII/CREB signalling pathway. Inhibitors of ASICs may serve as novel candidates for migraine therapy.

\section{Disclosure Statement}

No conflict of interests exists.

\section{References}

1 May A, Schulte LH: Chronic migraine: risk factors, mechanisms and treatment. Nat Rev Neurol 2016;12:455-464.

- Ferrari MD, Klever RR, Terwindt GM, Ayata C, van den Maagdenberg AM: Migraine pathophysiology: lessons from mouse models and human genetics. Lancet Neurol 2015;14:65-80.

-3 Oshinsky ML, Gomonchareonsiri S: Episodic dural stimulation in awake rats: a model for recurrent headache. Headache 2007;47:1026-1036.

-4 Wieseler J, Ellis A, Sprunger D, Brown K, McFadden A, Mahoney J, Rezvani N, Maier SF, Watkins LR: A novel method for modeling facial allodynia associated with migraine in awake and freely moving rats. J Neurosci Methods 2010;185:236-245.

-5 Yan J, Edelmayer RM, Wei X, De Felice M, Porreca F, Dussor G: Dural afferents express acid-sensing ion channels: a role for decreased meningeal pH in migraine headache. Pain 2011;152:106-113.

6 Yan J, Wei X, Bischoff C, Edelmayer RM, Dussor G: pH-evoked dural afferent signaling is mediated by ASIC3 and is sensitized by mast cell mediators. Headache 2013;53:1250-1261.

7 Pradhan AA, Smith ML, McGuire B, Tarash I, Evans CJ, Charles A: Characterization of a novel model of chronic migraine. Pain 2014;155:269-274.

-8 Huang D, Ren L, Qiu C-S, Liu P, Peterson J, Yanagawa Y, Cao Y-Q: Characterization of a mouse model of headache. Pain 2016;157:1744-1760. 


\section{Cellular Physiology Cell Physiol Biochem 2018;46:568-578 \begin{tabular}{l|l} 
DOI: 10.1159/000488624 & Ond Biochemistry 2018 The Author(s). Published by S. Karger AG, Basel \\
www.karger.com/cpb
\end{tabular} \\ Wang et al.: Increased Asics Expression in a Novel Mouse Model of Trigeminal Pain}

9 Malick A, Jakubowski M, Elmquist JK, Saper CB, Burstein R: A neurohistochemical blueprint for paininduced loss of appetite. Proc Natl Acad Sci U S A 2001;98:9930-9935.

10 Hoeger-Bement MK, Sluka KA: Phosphorylation of CREB and mechanical hyperalgesia is reversed by blockade of the cAMP pathway in a time-dependent manner after repeated intramuscular acid injections. J Neurosci 2003;23:5437-5445.

11 Burstein R, Yamamura H, Malick A, Strassman AM: Chemical stimulation of the intracranial dura induces enhanced responses to facial stimulation in brain stem trigeminal neurons. J Neurophysiol 1998;79:964982.

12 Durham PL, Masterson CG: Two mechanisms involved in trigeminal CGRP release: implications for migraine treatment. Headache 2013;53:67-80.

13 Albury CL, Stuart S, Haupt LM, Griffiths LR: Ion channelopathies and migraine pathogenesis. Mol Genet Genomics 2017;292:729-739.

14 Nakanishi M, Hata K, Nagayama T, Sakurai T, Nishisho T, Wakabayashi H, Hiraga T, Ebisu S, Yoneda T: Acid activation of Trpv1 leads to an up-regulation of calcitonin gene-related peptide expression in dorsal root ganglion neurons via the CaMK-CREB cascade: a potential mechanism of inflammatory pain. Mol Biol Cell 2010;21:2568-2577.

15 Wemmie JA, Taugher RJ, Kreple CJ: Acid-sensing ion channels in pain and disease. Nat Rev Neurosci 2013;14:461-471.

16 Deval E, Gasull X, Noel J, Salinas M, Baron A, Diochot S, Lingueglia E: Acid-sensing ion channels (ASICs): pharmacology and implication in pain. Pharmacol Ther 2010;128:549-558.

17 Bolay H, Reuter U, Dunn AK, Huang Z, Boas DA, Moskowitz MA: Intrinsic brain activity triggers trigeminal meningeal afferents in a migraine model. Nat Med 2002;8:136-142.

18 Shimada SG, LaMotte RH: Behavioral differentiation between itch and pain in mouse. Pain 2008;139:681687.

19 Holland PR, Akerman S, Andreou AP, Karsan N, Wemmie JA, Goadsby PJ: Acid-sensing ion channel 1: a novel therapeutic target for migraine with aura. Ann Neurol 2012;72:559-563.

20 Edelmayer RM, Vanderah TW, Majuta L, Zhang ET, Fioravanti B, De Felice M, Chichorro JG, Ossipov MH, King T, Lai J, Kori SH, Nelsen AC, Cannon KE, Heinricher MM, Porreca F: Medullary pain facilitating neurons mediate allodynia in headache-related pain. Ann Neurol 2009;65:184-193.

21 Deval E, Noel J, Lay N, Alloui A, Diochot S, Friend V, Jodar M, Lazdunski M, Lingueglia E: ASIC3, a sensor of acidic and primary inflammatory pain. EMBO J 2008;27:3047-3055.

22 Dussor G: ASICs as therapeutic targets for migraine. Neuropharmacology 2015;94:64-71.

23 Pietrobon D: Calcium channels and migraine. Biochim Biophys Acta 2013;1828:1655-1665.

24 Nair A, Simonetti M, Birsa N, Ferrari MD, van den Maagdenberg AM, Giniatullin R, Nistri A, Fabbretti E: Familial hemiplegic migraine Ca(v)2.1 channel mutation R192Q enhances ATP-gated P2X3 receptor activity of mouse sensory ganglion neurons mediating trigeminal pain. Mol Pain 2010;6:48.

25 Nimmrich V, Gross G: P/Q-type calcium channel modulators. Br J Pharmacol 2012;167:741-759.

-26 Fu H, Fang P, Zhou HY, Zhou J, Yu XW, Ni M, Zheng JY, Jin Y, Chen JG, Wang F, Hu ZL: Acid-sensing ion channels in trigeminal ganglion neurons innervating the orofacial region contribute to orofacial inflammatory pain. Clin Exp Pharmacol Physiol 2016;43:193-202. 